Bangladesh Journal of Neuroscience 2011; Vol. 27 (2) : 105-107

\title{
A Frontal Retrobulbar Mucocele with Lytic Lesion at Orbital Plate and Orbital Ridge, Rare Case Report
}

\author{
HARADHAN DEB NATH ${ }^{1}$, KANAK KANTI BARUA ${ }^{2}$
}

\begin{abstract}
Mucoceles can occurs when there is obstruction of drainage passage of mucosa of paranasal sinus. Some time it can cause pressure at eye ball and can cause proptosis. A old man admitted at Bangabandhu Sheikh Mujib Medical University with the complaints of right orbital swelling with proptosis of right eye ball. Also complaints of progressive visual bluring \& headache for some time. The mucocele was operated by frontal craniotomy and excised completely. As this is a ran case and so few an reported.
\end{abstract}

Key word: Mucocele, frontal, headache, proptosis, retrobulbar.

Introduction:

We reported the case as it is causes unusual submucosal extending with broken orbital plate and orbital ridge.

Mucocele are cystic lesions, with a wall formed by the mucous lining of the sinuses. They arise from the paranasal sinuses when there is an obstruction to the normal draining pathways. Long-standing obstruction leads to outward expansion of the sinus. The bony wall expand and may completely disappear, but the periosteal lining persists. They occur mostly in the frontal sinuses, followed by the ethmoid, maxillary and sphenoid sinuses ${ }^{1}$.

Extension of the mucoceles in the orbit can lead to proptosis that may or may not be accompanied by pain. They may cause others symptoms, such as visual disturbance, ocular palsy, and headache.Symptoms are seen mainly with posterior ethmoid and sphenoid mucoceles and may even be associated with neuralgias in the first and second trigeminal divisions. ${ }^{2} \mathrm{~A}$ history of recurrent sinus infection is occasionally elicited.

On computerized tomography scan, the sinus was enlarged and the surrounding cortex appears like a thin, dense line that may be absent in some areas. On MRI, the wall enhances with contrast, whereas the contents have a variable signal, depending on their age. Initially, they have a low protein content (low and high signal intensity on T1 weighted and
T2-weighted images, respectively). Subsequently, the protein concentration increase (T1-weighted signal intensity increase while T2-weighted signal intensity decrease more slowly, leading to a bright appearance on T1-weighted and T2-weighted images). With further evolution, the signal intensity drops on both sequences and eventually when the secretions desiccate, the signal becomes darker on both sequences ${ }^{1}$.

Treatment is surgical and consists of excising the mucosal lining of the cyst and restoring the drainage pathways of the occluded sinus. Recurrences may occur ${ }^{1}$.

\section{Case Report:}

A 60 years old male from remote area of Dhaka had been admitted at the Department of Neurosurgery at Bangabandhu Sheikh Mujib Medical University with the complaints slowly progressive swelling at right frontal resion and the swelling extended to forehead and supraorbital region. He also gave the history of dull headache and progressive visual blurring from same duration.

On examination his visual acquity was $6 / 60$, the eye ball was pushed downward and outward. The eye ball movement restricted upward. He also had conjunctival chemosis.

Other neurological examination were normal. On local examination there was proptosis of right eye ball, a

1. Assistant Professor, Department of Neurosurgery, Bangabandhu Sheikh Mujib Medical University, Dhaka.

2. Professor, Department of Neurosurgery, Department of Neurosurgery, Bangabandhu Sheikh Mujib Medical University, Dhaka. 
swelling at right frontal and supraorbital region and consistency was firm. Swelling was non tender, non pulsatile and there was no bruit or marmur over it. There was a bomy gap over the swelling.

All general parameters were normal. Contrast CT scan of head revealed non enhancing isodense lesion at the right frontal sinus extending also to right orbit. CT scan also showed bony destruction over orbital roof and posterior wall of frontal air sinus (Fig.-1). The swelling was operated with bicoronal scalp incision with right frontal craniotomy. There was a suckable tumor seem to be mucocle and was excised completely.
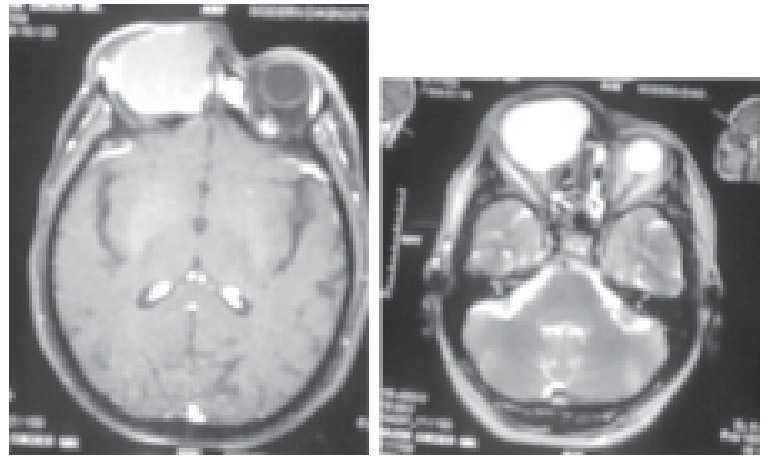

Fig.-1: Mucocele at the right orbital region

Sinus cavity was cleaned and packed with povidone iodine solution soaked spongastine and muscle patch and bone wax (Fig.-2 \& 3). Mini carnioplasty was done by autologus bone graft from iliac crest. Proper haemostasis was done. A drain was kept in situ which was removed at $1^{\text {st }}$ postoperative day and post operative period was uneventful (Fig.-4).

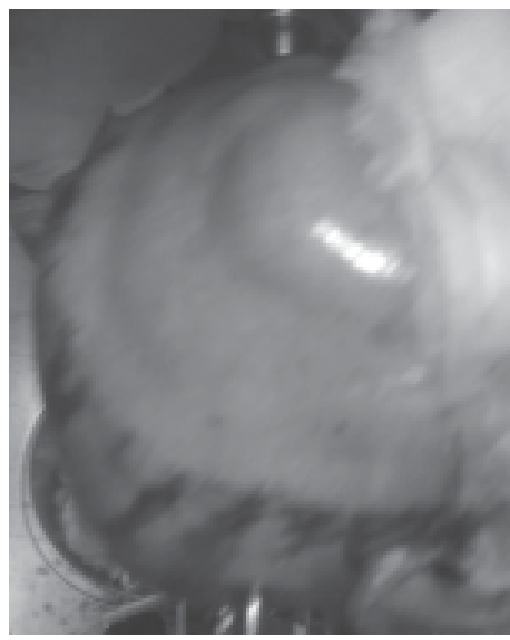

Fig.-2: Patient under anaesthesia with fronto-orbital mucocele

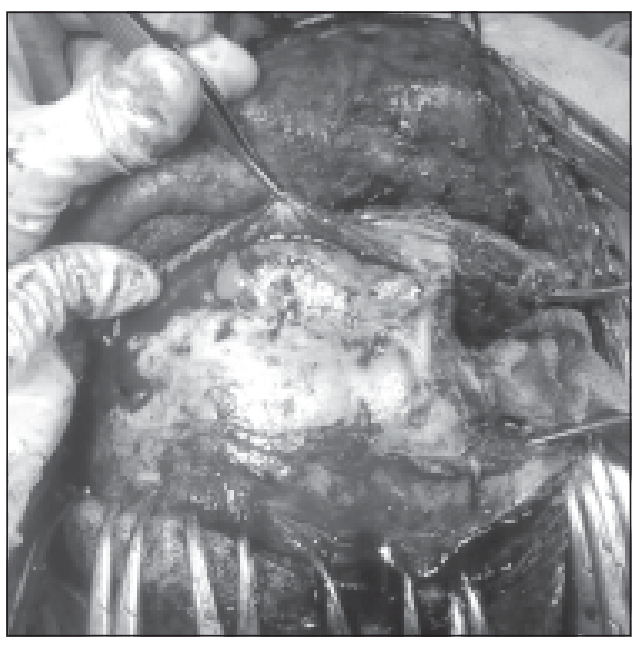

Fig.-3: Peroperative photograph of mucocele

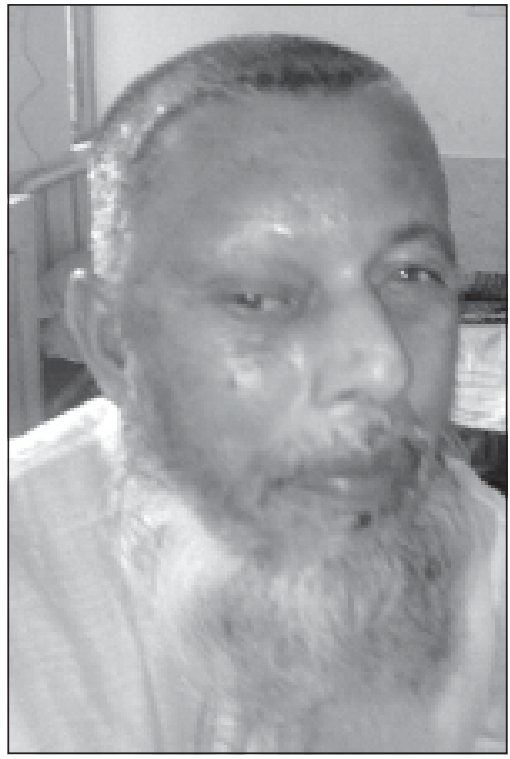

Fig.-4: Postoperative photograph

\section{Discussion:}

Mucoceles commonly involve the frontal sinus, maxillary sinus or anterior ethmoid sinus and rarely the posterior ethmoid or sphenoid sinus ${ }^{3}$. Frontal sinuses are the most common site for mucoceles and these can be frontoethmoidal or frontal only, but bilateral frontal involvement is rare $\mathrm{r}^{4,5}$. These lesions are usually observed in the fourth to sixth decade of life. No gender preference has been observed. Gradual distension, thinning and erosion of the bony wall of the sinus are caused by progressive accumulation of mucoid material. The 
mucocele can extend into the orbit or intracranial compartment by eroding the bony limits and producing bony defects. This may be the most likely pathogenesis in our case secondary to a deviated nasal septum. A ruptured intracranial mucocele can present with meningitis, meningoencephalitis, brain abscess, seizures or cerebrospinal fluid (CSF) fistula.

Mucoceles can present with diminution of vision, visual field defect, diplopia, orbital swelling, retroorbital pain, displacement of eye globe, ptosis, and proptosis ${ }^{6,7}$. Very rarely these lesions can present as a forehead swelling. To the best of authors' knowledge, only two cases with forehead swelling caused by giant frontal mucoceles have been reported in the western literature ${ }^{3,7}$. Tan CSH et al. ${ }^{7}$ reported a 33-year-old female presenting with blurring of the inferior visual field in the left eye, associated with periorbital swelling and a painless subcutaneous forehead mass. Akiyama $\mathrm{M}$ et al ${ }^{3}$ reported a similar case in a 57-year-old female, who presented with a history of an asymptomatic subcutaneous tumor on the forehead for threemonths. Borkar $\mathrm{S}$ described mucocele presenting with forehead subcutaneous mass ${ }^{8}$.

The swelling was $3 \mathrm{~cm}$ in diameter, on the mid- to left-side of forehead. Computerized tomography (CT) images showed a cystic mass demarcated from the subcutaneous area on the forehead caused by expansion of the frontal sinus by the mucocele. Computerized tomography (CT) is diagnostic for paranasal sinus mucocele ${ }^{9}$ but magnetic resonance imaging (MRI) is useful in infected cases to find out the exact intracranial extension and to rule out lesions such as chondromyxoma, cystic hypophyseal adenoma, schwannoma and retrobulbar cyst. These are rare but lesion which can easily be treated by surgery. More can be prevented by treatment of blockage of paranasal sinus drainage.

\section{Conclusion:}

We reported the case as it is benign lesion and can easily be removed by surgery. Endoscopic sinus surgery combined with transcranial surgery is advisable in case of giant frontal mucocele. Cranioplasty can done by autologus bone graft or methyl methacrylate.

\section{References:}

1. Curtin $\mathrm{H}$, Ribinov J. Extension to the orbit from paraorbital disease. The sinuses Radiol Clin North Am 1998; 36: 1201-13.

2. Maroon J, Kennerdell J, Brillman J. Tumors of the Orbit. In Wilkin RH, Rengashary SS (eds): Neurosurgery. New York, McGraw-Hill 1996; 1481-93.

3. Akiyama M, Inamoto N, Hashigucci K: Frontal mucocele presenting as a subcutaneous tumour on the forehead. Dermatology 1999; 3: 263-4.

4. Arrue P, Kany MT, Serrano E, et al. Mucoceles of the paranasal sinuses: uncommon location. J Laryngol Otol 1999; 112(9): 840-4.

5. Rao ABN: A Case of Bilateral Complicated Frontal Mucocele. Pract. Oto-Rhino-Laryngol 1966;28(1):117-20.

4. Hayasaka S, Shibasaki H, Sekimoto M et al. Ophthalmic complications in patients with paranasal sinus mucopyoceles. Ophthalmologica 1991; 203(2): 57-63.

6. Leventer DB, Linberg JV, Ellis B. Frontoethmoidal mucoceles causing bilateral chorioretinal folds. Arch Ophthalmol 2001; 119(6): 922-3.

7. Tan CSH, Yong VK, Yip LW et al. An unusual presentation of a giant frontal mucocele manifesting with a subcutaneous forehead mass. Ann Acad Med Singapore 2005; 34: 397-8.

8. Borkar S, Tripathi AK, Satarthee $G$ et al. Frontal mucocele presenting with forehead subcutaneous mass: An unsual presentation. Turkish Neurosurgery 2008; 18(2): 200-203.

9. Rao VM, Sharma D, Madan A: Imaging of frontal sinus disease: concepts, interpretation, and technology. Otolaryngol Clin North Am. 2001; 34(1): 23-9. 